\title{
Welcome to ISCT 2020 Paris Virtual
}

On behalf of the ISCT 2020 Organizing Committee, we would like to extend a warm welcome to ISCT 2020 Paris Virtual! As the leading global Society focused on the translation of cell and gene therapy, our mission remains as important as ever in the face of these uncertain times. The CGT field shows immense resilience as it rapidly evolves, and so must ISCT. We, together with our colleagues on the Organizing Committee and ISCT Head Office, are thus excited to showcase scientific excellence and innovation as we Accelerate Cell \& Gene Therapy Adoption: From Proof of Concept to Standard of Care through the inaugural ISCT Virtual Meeting. We are committed to bring you an unforgettable conference that will mix scientific excellence with pragmatism in bringing innovations to the market and to patients. This, of course, alongside friendship among peers across our global community.

Leveraging state-of-the-art virtual meeting technology, novel preclinical and translational science from 6 leading-edge areas of the CGT field will be showcased throughout the live broadcasted Plenary Sessions and associated Plenary Satellites. In particular, our Plenary Sessions will shine a spotlight on recent developments in the clinical application of MSC, CAR NK, and CAR T therapies, emerging gene-editing approaches for monogenetic and rare diseases, and the latest advancements in our understanding of extracellular vesicles and pluripotent cells as novel therapeutic agents. Unique to ISCT 2020 Paris Virtual, the program includes 2 feature sessions on the COVID-19 pandemic highlighting the current understanding of COVID-19 pathogenesis, individual reports from the front lines, supply chain issues, and current MSC and Immunotherapy treatment approaches.

A focal point of the annual meeting program remains the much anticipated Quality \& Operations and Strategies for Commercialization Tracks, while a variety of other on-demand workshops will offer delegates greater insight into emerging issues, breaking news, and application specific considerations in the field of translational cell \& gene therapy. Furthermore, ISCT 2020 witnessed a record-breaking number of abstract submissions which we are pleased to feature throughout the 48 hours of non-stop conference programming by way of both oral abstract presentations and an interactive E-Poster Hall.

Central to the execution of this high-caliber and comprehensive program is the valued support of our many industry partners who have sponsored the event; we are exceedingly thankful for their support. At the heart of the virtual meeting platform is our Exhibit Hall, where many of our partner organizations will be participating at customized exhibit booths. Be sure to visit the Exhibit Hall in between live broadcasted sessions and find the pulse of ISCT 2020's next generation networking opportunities. Make sure to also check out the Corporate Symposia, Tutorials and Global Showcase Presentations to be featured across both days of programming and stay up to date on the latest clinical research and product launches within the industry community.

Finally, we wish to extend our sincere gratitude to the dedicated and enthusiastic members who have contributed to the organization of this highly curated program. A huge thank you must also be extended to the incredible line-up of ISCT 2020 speakers who have graciously agreed to share their expertise and experiences during the virtual meeting. As a truly global Society we are excited to provide this highly interactive means of conferencing that will connect our members from all corners of the globe like never before. We hope you all enjoy your time together and look forward to a spectacular 2 days of digital age knowledge sharing, networking, and catching up with old friends.

Although we will not be able to share a coffee or your favorite drink with you in beautiful Paris, we would like to take this opportunity to reassure you that our community thrives with mutual respect and friendship. We will be waiting for the next editions of the ISCT Meeting in a conventional format, and in the meantime we wish you and your families, friends and teams to remain safe while facing these difficult times.

Sincerely,

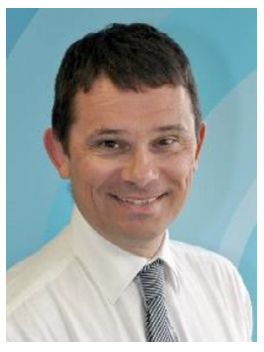

Christian Chabannon, MD, PhD ISCT 2020 Paris Co-Chair, Institut Paoli-Calmettes, FRA

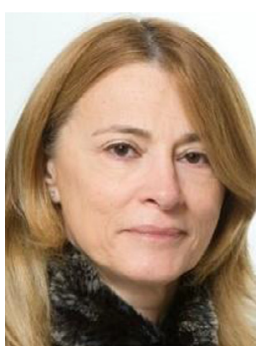

Rachele Ciccocioppo, MD ISCT 2020 Paris Co-Chair, University of Verona, ITA

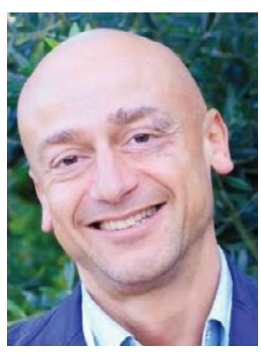

Ivan Martin, PhD ISCT 2020 Paris Co-Chair, University Hospital Basel, CHE 